Chinese Journal of Organic Chemistry

NOTE

\author{
垂穗石松中一个新的千层塔三萜

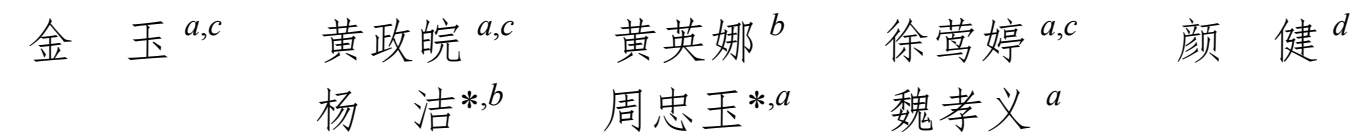 \\ $\left({ }^{a}\right.$ 中国科学院华南植物园 中国科学院植物资源保护与可持续利用重点实验室 \\ 广东省应用植物学重点实验室 广州 510650) \\ $\left({ }^{b}\right.$ 南方医科大学 广东省新药篮选重点实验室 广州市新发病毒防治药物研究重点实验室 广州 510515) \\ ( ${ }^{c}$ 中国科学院大学 北京 100049) \\ ( $d$ 华南农业大学资源环境学院 农业农村部华南热带农业环境重点实验室 广东省生态循环农业 \\ 重点实验室 广东省现代生态循环农业工程中心 广州 510642)
}

\begin{abstract}
摘要 利用多种色谱分离方法对垂穗石松的化学成分进行研究, 从其全株的乙醇提取物中分离得到 2 个化合物, 用 MS 和 NMR 等多种现代波谱技术确定了化合物的结构, 经鉴定分别为 3 $\beta, 14 \alpha, 15 \alpha, 21 \beta$-四羟基-15-(3'-甲氧基-4'-羟基苯甲 酰)-千层塔烷(1)和 16-酮基-3 $\beta, 21 \alpha$-二羟基-14-千层塔烯(2). 其中化合物 $\mathbf{1}$ 为新化合物, 2 个化合物均为首次从小石松属 植物分离.
\end{abstract}

关键词 垂穗石松; 千层塔三萜; 抗甲型流感病毒

\title{
A New Serratane Triterpenoid from Lycopodiella cernua
}

\author{
Jin, $\mathrm{Yu}^{a, c}$ \\ Huang, Zhengwan ${ }^{a, c}$ \\ Huang, Yingna ${ }^{b}$ \\ $\mathrm{Xu}$, Yingting ${ }^{a, c}$ \\ Yan, $\operatorname{Jian}^{d}$ \\ Yang, Jie $^{*, b} \quad$ Zhou, Zhongyu $^{*, a} \quad$ Wei, Xiaoyi $^{a}$
}

$\left({ }^{a}\right.$ Key Laboratory of Plant Resources Conservation and Sustainable Utilization \& Guangdong Provincial Key Laboratory of Applied Botany, South China Botanical Garden, Chinese Academy of Sciences, Guangzhou 510650)

$\left({ }^{b}\right.$ Guangdong Provincial Key Laboratory of New Drug Screening, Guangzhou Key Laboratory of Drug Research for Emerging

Virus Prevention and Treatment, School of Pharmaceutical Sciences, Southern Medical University, Guangzhou 510515)

( ${ }^{c}$ University of Chinese Academy of Sciences, Beijing 100049)

$\left({ }^{d}\right.$ Key Laboratory of Agro-environment in the Tropics, Ministry of Agriculture and Rural Affairs; Guangdong Provincial Key Laboratory of Eco-Circular Agriculture; Guangdong Engineering Research Centre for Modern Eco-Agriculture;

College of Natural Resources and Environment, South China Agricultural University, Guangzhou 510642)

\begin{abstract}
Two serratane triterpenoids, $3 \beta, 14 \alpha, 15 \alpha, 21 \beta$-tetrahydroxyserrat-15-(3'-methoxyl-4'-hydroxybenzoate) (1) and 16oxoserrat-14-en-3 $\beta, 21 \alpha$-diol (2), were isolated from the whole plant of Lycopodiella cernua. Compound 1 was a new one. The structures of these compounds were identified by analysis of spectral data. These two compounds were isolated from Lycopodiella genus for the first time.
\end{abstract}

Keywords Lycopodiella cernua; serratane triterpenoid; anti-influenza A virus

\section{Introduction}

Lycopodiella cernua (L.) Pic. Serm, belonging to the family Lycopodiaceae, has been used as a traditional Chinese folk medicine to treat rheumatism, quadriplegia and contusion for centuries. ${ }^{[1]}$ Palhinhaea cernua (L.) Franco $\&$ Vasc. is a synonym of Lycopodiella cernua. Previous phytochemical study of this plant species has led to the isolation of serratane triterpenoids, ${ }^{[2-6]}$ alkaloids, ${ }^{[7-13]}$ flavonoids, ${ }^{[14]}$ and neolignans. ${ }^{[4]}$ Serratane triterpenoids and

\footnotetext{
* Corresponding authors. E-mail: yj528@smu.edu.cn; zhouzhongyu@scbg.ac.cn Received April 3, 2020; revised April 27, 2020; published online May 19, 2020. Project supported by the National Natural Science Foundation of China (No. 31970376), the Youth Innovation Promotion Association, Chinese Academy of Sciences (No. 2016310), and the Guangzhou Science and Technology Program (No. 201904010134).

国家自然科学基金(No. 31970376)、中国科学院青年创新促进会会员(No. 2016310)、广州市科技计划(No. 201904010134)资助项目.
} 
lycopodium alkaloids are characteristic components of $L$. cernua plant. Serratane is a unique class of pentacyclic triterpenoids with a central seven-membered ring $\mathrm{C}$, which is rich in Lycopodiaceae family. Recently, the structural classification, biological activities and hypotheses about biosynthetic pathways of serratane-type triterpenes from Lycopodiaceae family was comprehensively reviewed. ${ }^{[15]}$ As part of our research on novel serratane triterpenoids from $L$. cernua ${ }^{[2,6]}$ two serratane triterpenoids, $3 \beta, 14 \alpha, 15 \alpha$, $21 \beta$-tetrahydroxyserrat-15-(3'-methoxyl-4'-hydroxybenzoate) (1), and 16-oxoserrat-14-en-3 $\beta, 21 \alpha$-diol (2), were isolated from the whole plant of Lycopodiella cernua (Figure 1). Compound 1 was a new one. Herein, we report the isolation, structural identification and anti-influenza A virus (IAV) activity of these two compounds.
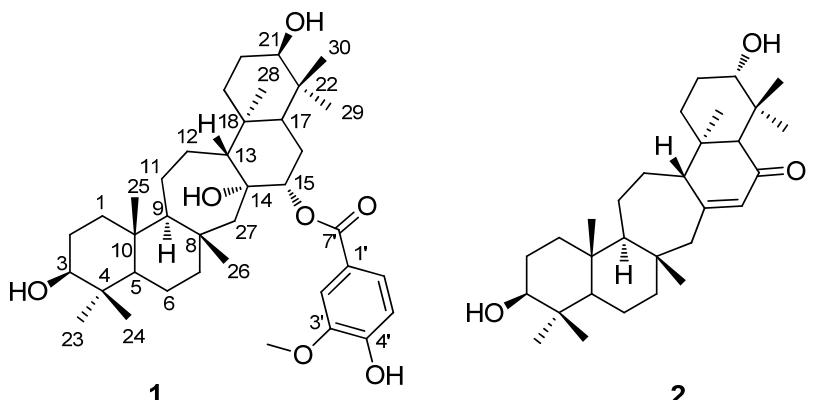

Figure 1 Structures of compounds 1 and 2

\section{Results and discussion}

Compound 1 was obtained as a white amorphous powder. The molecular formula of $\mathbf{1}$ was determined as $\mathrm{C}_{38} \mathrm{H}_{58} \mathrm{O}_{7}$ by positive HRESIMS $\left(\mathrm{m} / z[\mathrm{M}+\mathrm{H}]^{+}\right.$627.4247, calcd for $\left.\mathrm{C}_{38} \mathrm{H}_{59} \mathrm{O}_{7}: 627.4255\right)$ in combination with the ${ }^{13} \mathrm{C}$ and DEPT NMR analysis. The ${ }^{1} \mathrm{H}$ NMR spectrum (Table
1) displayed the signals for seven methyls at $\delta_{\mathrm{H}} 0.74(\mathrm{~s}$, $3 \mathrm{H}, \mathrm{H}-23$ ), 0.92 (s, 3H, H-24), 0.84 (s, 3H, H-25), 1.07 (s, $3 \mathrm{H}, \mathrm{H}-26), 1.02$ (s, 3H, H-28), 0.95 (s, 3H, H-29), 0.87 (s, $3 \mathrm{H}, \mathrm{H}-30)$, an oxygenated methyl at $\delta_{\mathrm{H}} 3.92$, three methines at $\delta_{\mathrm{H}} 3.11(\mathrm{dd}, J=11.5,4.8 \mathrm{~Hz}, 1 \mathrm{H}, \mathrm{H}-3), 3.36(\mathrm{t}$, $J=2.5 \mathrm{~Hz}, 1 \mathrm{H}, \mathrm{H}-21)$, and $4.94(\mathrm{dd}, J=11.3,4.6 \mathrm{~Hz}, 1 \mathrm{H}$, $\mathrm{H}-15)$, and a 1,3,4-trisubstituted benzene ring at $\delta_{\mathrm{H}} 7.68(\mathrm{~d}$, $\left.J=1.9 \mathrm{~Hz}, 1 \mathrm{H}, \mathrm{H}-2^{\prime}\right), 6.86$ (d, $\left.J=8.2 \mathrm{~Hz}, 1 \mathrm{H}, \mathrm{H}-5^{\prime}\right), 7.70$ (dd, $J=8.2,1.9 \mathrm{~Hz}, 1 \mathrm{H}, \mathrm{H}-6$ ). The ${ }^{13} \mathrm{C}$ and DEPT NMR spectra suggested the presence of ten quaternary carbons, ten methines, ten methylenes, and eight methyls. The ${ }^{13} \mathrm{C}$ NMR spectra (DEPT) showed signal at $\delta_{\mathrm{C}} 55.8$ for a methylene, which was characteristic signal of C-27 in serratane-type triterpenoid. ${ }^{[6]}$ By detailed analysis of the HMBC spectrum of compound 1 and comparison of its NMR data with those of $3 \beta, 14 \alpha, 15 \alpha, 21 \alpha$-tetrahydroxyserrat-3-(3'-methoxyl-4'-hydroxybenzoate), ${ }^{[2]}$ the serratane triterpenoid skeleton was assigned as 3,14,15,21-tetrahydroxyserratane. Except 30 carbons signals for a serratane triterpenoid, the remaining signals in the ${ }^{1} \mathrm{H}$ NMR and ${ }^{13} \mathrm{C}$ NMR spectra of compound 1 indicated the presence of a 3'-methoxy-4'hydroxybenzoate group through comparing corresponding NMR data with $3 \beta, 14 \alpha, 15 \alpha, 21 \alpha$-tetrahydroxyserrat-3-(3'methoxyl-4'-hydroxybenzoate). ${ }^{[2]}$ The ester linkage between C-15 of serratane triterpenoid and C-7' of 3'-methoxy-4'-hydroxybenzoate was established by heteronuclear multiple bond correlation (HMBC) correlation (Figure 2) from $\mathrm{H}-15\left(\delta_{\mathrm{H}} 4.94\right)$ to $\mathrm{C}-7^{\prime}\left(\delta_{\mathrm{C}} 168.0\right)$. The big coupling constant $(J=11.5 \mathrm{~Hz})$ of $\mathrm{H}-3$ indicated ax-ax coupling with axial $\mathrm{H}-2$, while the small coupling constant $(J=4.8$ $\mathrm{Hz}$ ) of $\mathrm{H}-3$ indicated ax-eq coupling with equatorial $\mathrm{H}-2$. Therefore, $\mathrm{H}-3$ was at axial bond and was $\alpha$-orientated. ${ }^{[3]}$ In the same way, H-21 appeared to be equatorial (on the $\alpha$-face) according to its small coupling constants of 2.5

Table $1{ }^{1} \mathrm{H}$ NMR and ${ }^{13} \mathrm{C}$ NMR (600 and $150 \mathrm{MHz}$, resp.) data for $\mathbf{1}$ in $\mathrm{CD}_{3} \mathrm{OD}$

\begin{tabular}{clc||clc}
\hline No. & \multicolumn{1}{c||}{$\delta_{\mathrm{H}}$} & $\delta_{\mathrm{C}}$ & No. & & $\delta_{\mathrm{H}}$ \\
\hline 1 & $1.78 \sim 1.80(\mathrm{~m}, 1 \mathrm{H}), 0.99 \sim 1.01(\mathrm{~m}, 1 \mathrm{H})$ & 39.9 & 20 & $2.00 \sim 2.02(\mathrm{~m}, 1 \mathrm{H}), 1.56 \sim 1.58(\mathrm{~m}, 1 \mathrm{H})$ & 26.2 \\
2 & $1.58 \sim 1.60(\mathrm{~m}, 2 \mathrm{H})$ & 28.2 & 21 & $3.36(\mathrm{t}, 2.5,1 \mathrm{H})$ & 76.7 \\
3 & $3.11(\mathrm{dd}, 11.5,4.8,1 \mathrm{H})$ & 79.7 & 22 & & 38.7 \\
4 & & 40.0 & 23 & $0.92(\mathrm{~s}, 3 \mathrm{H})$ & 28.6 \\
5 & $0.78(\mathrm{dd}, 9.1,5.2,1 \mathrm{H})$ & 57.1 & 24 & $0.74(\mathrm{~s}, 3 \mathrm{H})$ & 16.1 \\
6 & $1.44 \sim 1.46(\mathrm{~m}, 2 \mathrm{H})$ & 19.6 & 25 & $0.84(\mathrm{~s}, 3 \mathrm{H})$ & 16.6 \\
7 & $1.24 \sim 1.26(\mathrm{~m}, 2 \mathrm{H})$ & 46.7 & 26 & $1.07(\mathrm{~s}, 3 \mathrm{H})$ & 21.4 \\
8 & & 38.6 & 27 & $\alpha, 1.36(\mathrm{~d}, 15.5,1 \mathrm{H}), \beta, 1.66(\mathrm{~d}, 15.5,1 \mathrm{H})$ & 55.8 \\
9 & $1.13(\mathrm{dd}, 11.2,2.2,1 \mathrm{H})$ & 63.2 & 28 & $1.02(\mathrm{~s}, 3 \mathrm{H})$ & 16.5 \\
10 & & 39.3 & 29 & $0.87(\mathrm{~s}, 3 \mathrm{H})$ & 22.6 \\
11 & $1.92 \sim 1.94(\mathrm{~m}, 1 \mathrm{H}), 1.22 \sim 1.24(\mathrm{~m}, 1 \mathrm{H})$ & 27.8 & 30 & $0.95(\mathrm{~s}, 3 \mathrm{H})$ & 29.0 \\
12 & $1.72 \sim 1.74(\mathrm{~m}, 1 \mathrm{H}), 1.47 \sim 1.49(\mathrm{~m}, 1 \mathrm{H})$ & 24.6 & $1^{\prime}$ & & 122.9 \\
13 & $1.37 \sim 1.39(\mathrm{~m}, 1 \mathrm{H})$ & 59.7 & $2^{\prime}$ & $7.68(\mathrm{~d}, 1.9,1 \mathrm{H})$ & 114.0 \\
14 & & 78.1 & $3^{\prime}$ & & 148.8 \\
15 & $4.94(\mathrm{dd}, 11.3,4.6,1 \mathrm{H})$ & 81.0 & $4^{\prime}$ & & 152.9 \\
16 & $1.89 \sim 1.91(\mathrm{~m}, 1 \mathrm{H}), 1.68 \sim 1.70(\mathrm{~m}, 1 \mathrm{H})$ & 25.3 & $5^{\prime}$ & $6.86(\mathrm{~d}, 8.2,1 \mathrm{H})$ & 116.0 \\
17 & $1.57 \sim 1.59(\mathrm{~m}, 1 \mathrm{H})$ & 47.6 & $6^{\prime}$ & $7.70(\mathrm{dd}, 8.2,1.9,1 \mathrm{H})$ & 125.5 \\
18 & & 39.8 & $7^{\prime}$ & & 168.0 \\
19 & $1.49 \sim 1.51(\mathrm{~m}, 1 \mathrm{H}), 1.40 \sim 1.42(\mathrm{~m}, 1 \mathrm{H})$ & 33.7 & $3^{\prime}-\mathrm{OCH}_{3}$ & $3.92(\mathrm{~s}, 3 \mathrm{H})$ & 56.6 \\
\hline
\end{tabular}




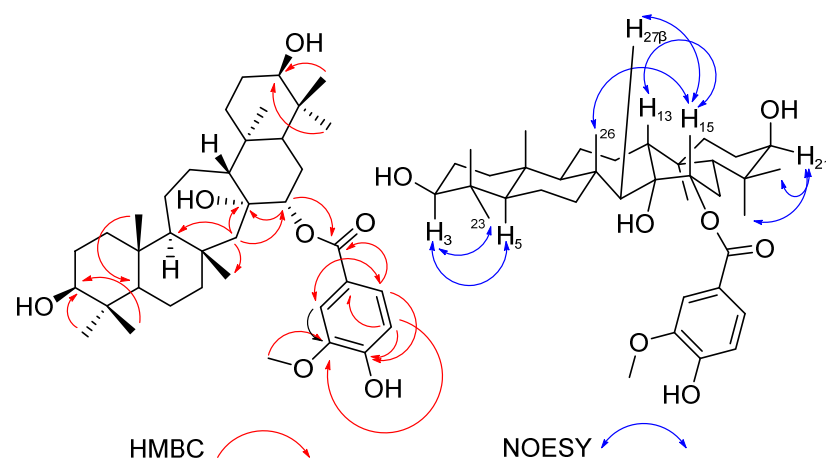

Figure 2 Significant HMBC (from $\mathrm{H}$ to $\mathrm{C}$ ) and NOESY correlations of compound $\mathbf{1}$

$\mathrm{Hz} .{ }^{[3]}$ The nuclear overhauser effect spectroscopy (NOESY) correlation (Figure 2) between $\mathrm{H}-15$ and $\mathrm{CH}_{3}-26 \beta$, confirmed the $\beta$ configuration of $\mathrm{H}-15$. According to literature, ${ }^{[16]}$ osmolation of lycernuic acid A diacetate gave two isomeric glycols. The minor glycol was proven to be $14 \alpha, 15 \alpha$-diol, while the major one to be $14 \beta, 15 \beta$-diol. The chemical shifts of C-17 in $14 \alpha, 15 \alpha$-diol and $14 \beta, 15 \beta$-diol were at $\delta_{\mathrm{C}} 48.0$ and 40.9 , respectively. Compound 1 had a chemical shift of C-17 at $\delta_{\mathrm{C}} 47.6$, which indicated $14 \alpha, 15 \alpha$ configurations of two hydroxyl groups in compound $\mathbf{1}$. Therefore, compound 1 was elucidated as $3 \beta, 14 \alpha, 15 \alpha$, $21 \beta$-tetrahydroxyserrat-15-(3'-methoxyl-4'-hydroxybenzoate) as shown in Figure 1.

The structure of known compound $\mathbf{2}$ was identified as 16-oxoserrat-14-en-3 $\beta, 21 \alpha$-diol (2) by interpretation of its spectroscopic data, as well as by comparison with reported values. $^{[17]}$

Compounds $\mathbf{1}$ and $\mathbf{2}$ were evaluated for their anti-IAV activity against A/WSN/33 (H1N1). Preliminary antiviral screening showed that compounds $\mathbf{1}$ and $\mathbf{2}$ displayed 9\% and $36 \%$ inhibition activity at $50 \mu \mathrm{mol} \cdot \mathrm{L}^{-1}$, respectively.

\section{Conclusions}

In conclusion, two serratane triterpenoids, $3 \beta, 14 \alpha, 15 \alpha, 21 \beta$ tetrahydroxyserrat-15-(3'-methoxyl-4'-hydroxybenzoate) (1) and 16-oxoserrat-14-en-3 $\beta, 21 \alpha$-diol (2), were isolated from the whole plant of Lycopodiella cernua. Compound $\mathbf{1}$ was a new one. These two compounds were isolated from Lycopodiella genus for the first time.

\section{Experimental section}

\subsection{General}

Optical rotations were measured on a Perkin-Elmer Model 341 polarimeter (Perkin-Elmer, Inc., Waltham, MA). UV spectra were recorded on a Perkin-Elmer Lambda $650 \mathrm{UV}-\mathrm{Vis}$ spectrophotometer. Nuclear magnetic resonance (NMR) spectra were recorded on a Bruker DRX-400 NMR spectrometer (Bruker Biospin Gmbh, Rheistetten, Germany) and a Bruker AVANCE 600 instrument. ESIMS was collected on an MDS SCIEX API 2000 LC/MS/MS instrument. HRESI-MS was acquired on a time-of-flight mass spectrometer (Bruker maxis 4G).
Medium pressure liquid chromatography (MPLC) and preparative HPLC were carried out on a CXTH P3000 instrument (Beijing Chuang Xin Tong Heng Science and Technology Co., Ltd, Beijing, China) equipped with a UV 3000 UV-vis Detector. A Fuji-C18 column $(10 \mu \mathrm{m}, 30$ $\mathrm{mm} \times 250 \mathrm{~mm}$ ) was used for preparative HPLC. For column chromatography, silica gel $(80 \sim 100$ mesh and $200 \sim$ 300 mesh Qingdao Haiyang Chemical Co., Qingdao, China), and Sephadex LH-20 (Pharmacia Fine Chemical Co., Ltd., Oppsala, Sweden) were performed. Thin-layer chromatography (TLC) was conducted on pre-coated silica gel plates $\left(\mathrm{HSGF}_{254}\right.$, Yantai Jiang you Silica Gel Development Co., Ltd., Yantai, China) and spot detection was performed by spraying $10 \% \mathrm{H}_{2} \mathrm{SO}_{4}$ in ethanol, followed by heating.

\subsection{Plant material}

The information of L. cernua plant material was published in our previous study. ${ }^{[2]}$

\subsection{Extraction and isolation}

The extraction procedure was previously published, ${ }^{[2]}$ and six serratane triterpenoids were isolated in our previous study. ${ }^{[2]}$ The remaining subfractions of $L$. cernua extracts were isolated in this study. Subfractions A and B were obtained from EtOAc fraction of $L$. cernua extracts after silica gel column chromatography and medium pressure liquid chromatography (MPLC). Subfraction A was applied to Sephadex LH-20 column chromatography $\left(\mathrm{CHCl}_{3}-\mathrm{MeOH}, V: V=1: 4\right)$, and followed by preparative HPLC $\left(80 \%\right.$ aqueous $\mathrm{CH}_{3} \mathrm{OH}, 10 \mathrm{~mL} / \mathrm{min}, R_{\mathrm{t}}=84$ $\min )$ to afford compound $1(11 \mathrm{mg})$. Subfraction B was separated by Sephadex LH-20 column chromatography $\left(\mathrm{CHCl}_{3}-\mathrm{MeOH}, V: V=1: 4\right)$ to give subfractions $\mathrm{B} 1$ and B2. Compound $2^{[17]}(5 \mathrm{mg})$ was obtained from subfraction B2 by preparative HPLC ( $84 \%$ aqueous $\mathrm{CH}_{3} \mathrm{OH}, 10$ $\mathrm{mL} / \mathrm{min}, R_{\mathrm{t}}=84 \mathrm{~min}$ ).

$3 \beta, 14 \alpha, 15 \alpha, 21 \beta$-tetrahydroxyserrat-15-(3'-methoxyl-4'hydroxybenzoate) (1): White amorphous powder, m.p. $220 \sim 222{ }^{\circ} \mathrm{C} ;[\alpha]_{\mathrm{D}}^{20}-12.61\left(c 0.33, \mathrm{CH}_{3} \mathrm{OH}\right)$; UV $\left(\mathrm{CH}_{3}-\right.$ OH) $\lambda_{\max }\left[\log \varepsilon /\left(\mathrm{L} \cdot \mathrm{mol}^{-1} \cdot \mathrm{cm}^{-1}\right)\right]: 217.02(3.16), 224.94$ (3.30), 263.32 (2.98), 293.53 (1.72) nm; ${ }^{1} \mathrm{H}$ NMR and ${ }^{13} \mathrm{C}$ NMR see Table 1. HRESIMS (positive) calcd for $\mathrm{C}_{38} \mathrm{H}_{59} \mathrm{O}_{7}$ $[\mathrm{M}+\mathrm{H}]^{+}$627.4255, found 627.4247 .

\subsection{Antiviral activity}

Two isolated compounds $\mathbf{1}$ and $\mathbf{2}$ were evaluated for their anti-IAV activities according to the previously reported 3-(4,5-dimethylthiazol-2yl)-2,5-diphenyltetrazolium bromide (MTT) assay, ${ }^{[18]}$ using zanamivir $\left(200 \mathrm{nmol} \cdot \mathrm{L}^{-1}\right)$ as a positive control. Test compounds were preliminary screened at two concentrations of 50 and $10 \mu \mathrm{mol} \cdot \mathrm{L}^{-1}$, and those which had more than $50 \%$ inhibition of influenza virus infection activity were further experimented for cytotoxicity and $\mathrm{IC}_{50}$ evaluation. In detail, Madin Derby canine kidney (MDCK) cells were cultured overnight in Dulbecco's modified Eagle's medium (DMEM) supplemented with $10 \%$ fetal bovine serum and $1 \%$ penicillin/streptomycin in 96 well plates. A/WSN/33 (H1N1) in- 
fluenza virus $\left(100 \mathrm{TCID}_{50}\right)$, which was propagated from 8 -day-old chicken embryos, was added and incubated for 1 $\mathrm{h}$. Then the supernatant was removed and washed with phosphate-buffered saline (PBS), and MDCK cells infected with influenza virus were exposed to $200 \mu \mathrm{L}$ of compounds 1 and 2 at 50 or $10 \mu \mathrm{mol} \cdot \mathrm{L}^{-1}$. After $48 \mathrm{~h}$ of incubation, $100 \mu \mathrm{L}$ of MTT solution, which was diluted by the medium to $0.5 \mathrm{mg} / \mathrm{mL}$, was added and retained at $37{ }^{\circ} \mathrm{C}$ for $4 \mathrm{~h}$. Then the supernatant was removed, followed by the addition of $150 \mu \mathrm{L}$ of dimethyl sulfoxide (DMSO) to dissolve the formazan product. The optical density for each well was measured on the Tecan Genios Pro microplate reader (Bedford, MA, USA) at $570 \mathrm{~nm}$.

Supporting Information ${ }^{1} \mathrm{H}$ NMR, ${ }^{13} \mathrm{C}$ NMR, HSQC, HMBC, and NOESY spectra of compounds 1 and 2. The Supporting Information is available free of charge via the Internet at http://sioc-journal.cn.

\section{References}

[1] Xiao, P. G. Modern Chinese Material Medica, Chemical Industry Press, Beijing, 2002, p. 135 (in Chinese). (肖培根, 新编中药志第三卷, 化学工业出版社, 北京, 2002, p. 135.)

[2] Yan, J.; Zhou, Z.-Y.; Zhang, M.; Wang, J.; Tan, J.-W. Planta Med. 2012, 78, 1387.

[3] Wei, J. J.; Wang, W. Q.; Song, W. B.; Xuan, L. J. Fitoterapia 2018 , 127,151 .
[4] Li, J.; Xu, P. S.; Tan, L. H.; Zou, Z. X.; Wang, Y. K.; Long, H. P.; Zhou, G.; Li, G.; Xu, K. P.; Tan, G. S. Fitoterapia 2017, 119, 45.

[5] Liang, L. F.; Chen, Q. H.; Xu, J. H.; Liu, T.; Song, X. N.; Chen, H. Y.; Chen, H. Chem. Nat. Compd. 2019, 55, 759.

[6] Yan, J.; Sun, L.-R.; Zhang, X.-M.; Li, Z.-R.; Zhou, L.; Qiu, M.-H. Chem. Pharm. Bull. 2009, 57, 1381.

[7] Zhao, F. W.; Sun, Q. Y.; Yang, F. M.; Hu, G. W.; Luo, J. F.; Tang, G. H.; Wang, Y. H.; Long, C. L. Org. Lett. 2010, 12, 3922.

[8] Zhao, F. W.; Sun, Q. Y.; Yang, F. M.; Luo, J. F.; Hu, G. W.; Liu, F.; Wang, Y. H.; Long, C. L. J. Brazil. Chem. Soc. 2012, 23, 349.

[9] Zhang, D. B.; Chen, J. J.; Zhang, L.; Song, Q. Y.; Gao, K. Phytochem. Lett. 2014, 10, 76.

[10] Tang, Y.; Xiong, J.; Zou, Y. K.; Zhang, H. Y.; Hu, J. F. Phytochemistry 2016, 131, 130.

[11] Tang, Y.; Xiong, J.; Zou, Y.; Nay, B.; Wang, L. J.; Hu, J. F. Chin. Chem. Lett. 2016, 27, 969.

[12] Dong, L. B.; Yang, J.; He, J.; Luo, H. R.; Wu, X. D.; Deng, X.; Peng, L. Y.; Cheng, X.; Zhao, Q. S. Chem. Commun. 2012, 48, 9038.

[13] Dong, L. B.; Gao, X.; Liu, F.; He, J.; Wu, X. D.; Li, Y.; Zhao, Q. S. Org. Lett. 2013, 15, 3570.

[14] Jiao, R. H.; Ge, H. M.; Shi, D. H.; Tan, R. X. J. Nat. Prod. 2006, 69, 1089.

[15] Boonya-udtayan, S.; Thasana, N.; Jarussophon, N.; Ruchirawat, S. Fitoterapia 2019, 136, 104181.

[16] Zhang, Z. Z.; ElSohly, H. N.; Jacob, M. R.; Pasco, D. S.; Walker, L. A.; Clark, A. M. J. Nat. Prod. 2002, 65, 979.

[17] Yang, G.; Zang, Y.; Hu, C.; Xiong, J.; Hu, J. Chin. Trad. Herbal Drugs 2014, 45, 3524 (in Chinese). (杨国勋，藏毅，胡长玲，熊娟，胡金锋，中草药, 2014, 45, 3524.)

[18] Luo, X.; Yang, J.; Chen, F.; Lin, X.; Chen, C.; Zhou, X.; Liu, S.; Liu, Y. Front. Chem. 2018, 6, 282 